\title{
GLUON SPIN IN THE NUCLEON *
}

\author{
R. L. Jaffe \\ Center for Theoretical Physics \\ Laboratory for Nuclear Science \\ and Department of Physics \\ Massachusetts Institute of Technology \\ Cambridge, Massachusetts 02139 \\ and \\ Lyman Physics Laboratory \\ Harvard University \\ Cambridge, Massachusetts 02138
}

(MIT-CTP-2466 ${ }^{\dagger}$ HUTP-95/A034 To be published in Physics Letters B October 1995)

\begin{abstract}
We study the operator description of the gluon spin contribution $(\Gamma)$ to the nucleon's spin as it is measured in deep inelastic processes. $\Gamma$ can be related to the forward matrix element of a local gluon operator in $A^{+}=0$ gauge. In quark models the nucleon contains ambient color electric and magnetic fields. The latter are thought to be responsible for spin splittings among the light baryons. We show that these fields give rise to a significant negative contribution to $\Gamma$ at the quark model renormalization scale, $\mu_{0}^{2}$. The nonAbelian character of QCD is responsible for the sign of $\Gamma$. In a generic nonrelativistic quark model $\Gamma_{N Q M}=-\frac{8}{9} \frac{\alpha_{N Q M}}{m_{q}}\left\langle\frac{1}{r}\right\rangle$, in the bag model $\Gamma_{b a g}=$ $-.1 \alpha_{b a g}$. These correspond to $\Gamma_{N Q M} \approx-0.7$ and $\Gamma_{b a g} \approx-0.4$ at $\alpha_{Q C D} \approx 1.0$.
\end{abstract}

\footnotetext{
${ }^{\dagger}$ Revised Version

${ }^{*}$ This work is supported in part by funds provided by the U.S. Department of Energy (D.O.E.) under cooperative agreement \#DF-FC02-94ER40818 and \#DE-FG02-92ER40702 and in part by funds provided by the National Science Foundation under grant \# PHY 92-18167
} 


\section{INTRODUCTION}

Since quark spin accounts for only a small fraction, $\Sigma \approx 0.3$, of the nucleon's spin 1 . wonders where the rest of the spin resides. Sehgal [3 pointed out long ago that experimental data on hyperon $\beta$-decays and the assumption that any strange quarks in the nucleon are unpolarized $\|$ requires $\Sigma \approx 0.6$ and he suggested that quark orbital angular momentum $\left(L_{Q}\right)$ - expected in relativistic quark models - was a likely candidate.

More recently, the possibility that gluons may carry a significant fraction of the nucleon's spin has been raised and debated. 58 Much debate has centered on the possibility that a gluonic contribution may "contaminate" the axial charge sum rules used to extract $\Sigma$ from polarized lepton scattering data, leading some to speculate that $\Gamma$ is large and positive. Whatever their impact on axial charges, there is no doubt that gluon spin $(\Gamma)$ and orbital angular momentum $\left(L_{G}\right)$ can contribute to the nucleon spin. In QCD the nucleon's total angular momentum can be written as

$$
\frac{1}{2}=L_{Q}+L_{G}+\frac{1}{2} \Sigma+\Gamma
$$

The definition of $\Sigma$ and its relation to the triangle anomaly were explored shortly after the publication of the SMC data. 5 However, the definitions of the other terms and their relation to local operators have received less attention. No one knows how to measure $L_{Q}$ and $L_{G} . \Gamma$ is defined as the integral of the polarized gluon distribution,

$$
\Gamma\left(Q^{2}\right)=\int_{0}^{1} d x \Delta g\left(x, Q^{2}\right)=\int_{0}^{1} d x\left(g_{\uparrow}\left(x, Q^{2}\right)-g_{\downarrow}\left(x, Q^{2}\right)\right)
$$

in the sense of a parton model sum rule.

The purpose of this Letter is to study $\Gamma$. First we review the definition of $\Delta g$ in terms of gauge invariant operators. 19 Next we integrate $\Delta g$, and specialize to $A^{+}=0$ gauge, where $\Gamma$ can be related to the matrix element of a local gluon operator. We discuss the gauge dependence of $\Gamma$ and its relation to the spin generators obtained via Noether's theorem. We conclude that $\Gamma$ is given by the matrix element of products of gluon vector potentials and field strengths in the nucleon rest frame and in $A^{+}=0$ gauge,

$$
\Gamma\left(Q^{2}\right)=\frac{1}{2 M}\left\langle\hat{e}_{3}\left|2 \operatorname{Tr}\left\{(\vec{E} \times \vec{A})^{3}+\vec{A}_{\perp} \cdot \vec{B}_{\perp}\right\}\right|_{Q^{2}} \mid \hat{e}_{3}\right\rangle
$$

where $\perp$ refers to the directions transverse to the $\hat{e}_{3}$ axis defined by the target spin, and $Q^{2}$ is the renormalization scale of the operators.

Eq. (3) is amenable to evaluation in constituent quark models. Just as quark models make predictions for $\Sigma$ which are tied to the phenomenology of $\beta$-decay axial currents, so too they make predictions for $\Gamma$, tied to the phenomenology of baryon mass differences. Quark models claim that baryon spin splittings (e.g. $M_{\Delta}-M_{N}$ ) originate in lowest order

\footnotetext{
${ }^{1}$ An earlier version of this paper contained an incorrect version of this equation which was incorrectly claimed to be gauge invariant. The author wishes to thank Ian Balitsky and Xiangdong Ji for raising questions of gauge invariance and for helpful discussions on other issues.
} 
exchange of transverse (magnetic) gluons. The predictions agree well with experiment, and the presentations have found their way into textbooks. [1] [1] Known as "color magnetism", this effect implicitly requires ambient color magnetic fields within hadrons. The model calculations are performed to lowest non-trivial order in $\alpha_{Q C D}$, where the gluons behave like eight Abelian vector fields coupled to color. The spin dependent piece of the Born graph for gluon exchange between quarks $i$ and $j$ can be rewritten as

$$
\Delta M=-\sum_{i>j} \sum_{a=1}^{8} \int d^{3} x \vec{B}_{i}^{a}(\vec{x}) \cdot \vec{B}_{j}^{a}(\vec{x})
$$

just as in electrodynamics. Thus the baryons contain color magnetic (and electric) fields which may carry spin angular momentum. The sign of $\Gamma$ is correlated with the sign of baryon spin splittings and originates in the non-abelian character of QCD forces. If quark spin forces were abelian, the $\Delta$ would be lighter than the nucleon and $\Gamma$ would be positive. The magnitude of $\Gamma$ depends on details of quark model wavefunctions and on the renormaliation scale assigned to the quark model calculations. However the model's prediction of the $\Delta-N$ mass difference constrains the size of $\Gamma$ to some extent.

A generic non-relativistic quark model (NQM) yields $\Gamma_{N Q M}=-\frac{8}{9} \frac{\alpha_{N Q M}}{m_{q}}\left\langle\frac{1}{r}\right\rangle$; the standard bag model yields $\Gamma_{b a g}=-0.1 \alpha_{b a g}$. Note that $2 \Gamma$ is the gluon spin fraction of the nucleon spin. We hesitate to assign a specific value to $\alpha$ for fear the resulting number will be taken too seriously. On the other hand the model parameters are constrained to some extent by the magnitude of baryon spin splittings making a numerical estimate possible. In the non-relativistic quark model the $N-\Delta$ splitting is $\Delta M=\frac{8 \pi \alpha_{N Q M}}{3 m_{q}^{2}}\left\langle\delta^{3}(\vec{r})\right\rangle$. If, for example, we choose Gaussian wave-functions scaled to reproduce the proton's charge radius, and a quark mass $m_{q} \approx M_{N} / 3$ to obtain approximately correct magnetic moments, then $\Delta M \approx 0.35 \alpha_{N Q M} M_{N}$, whence $\alpha_{N Q M} \approx 0.9$. With this value of $\alpha_{N Q M}$ we find $\Gamma_{N Q M} \approx-0.8$. In the bag model, $\alpha_{b a g} \approx 2$ in order to fit baryon mass differences, whence $\Gamma_{b a g} \approx-0.2$.

Note that these estimates apply at the quark model renormalization scale. To obtain a prediction for $Q^{2} \sim 2-10 \mathrm{GeV}^{2}$ relevant to experiment, it is necessary consider the evolution of $\Gamma$ with renormalization scale. It has long been known that $\Gamma$ evolves homogeneously, and that the quantity $\alpha\left(Q^{2}\right) \Gamma\left(Q^{2}\right)$ is a renormalization group invariant to leading order. [2] Therefore the sign of $\Gamma$ should be a reliable prediction of the models. If we take the numerical estimates seriously, evolution will tend to bring the two predictions closer together because the bag estimate will be multiplied by a larger factor of $\alpha\left(\mu_{0}^{2}\right) / \alpha\left(Q^{2}\right)$. For example, at $\alpha_{Q C D} \approx 1, \Gamma_{b a g} \approx-0.4$, and $\Gamma_{N Q M} \approx-0.7$.

In the next section we introduce and analyze the operator measure of gluon spin. We discuss gauge invariance. We show that the operator we evaluate in the rest frame is the same one measured by integrating the gluon helicity asymmetry $\Delta g\left(x, Q^{2}\right)$. In Section 3 , we estimate the magnitude of $\Gamma$ in simple models. In Section 4 we discuss the reliability of our calculation and and mention further applications.

\section{THE OPERATOR DESCRIPTION OF GLUON SPIN}

Experimenters will not measure $\left\langle(\vec{E} \times \vec{A})^{3}+\vec{A}_{\perp} \cdot \vec{B}_{\perp}\right\rangle$ directly. Instead they will measure the polarized gluon distribution function, $\Delta g\left(x, Q^{2}\right)$, in deep inelastic lepton scattering. $\Delta g$ 
measures the probability to find a gluon with its helicity parallel to the nucleon's helicity minus the probability to find it antiparallel. The integral of $\Delta g$ measures $\Gamma$ and therefore will tell us which local operator, if any, is to be associated with the gluon spin. We begin with the operator representation of the polarized gluon distribution function,

$$
\begin{aligned}
\Delta g\left(x, Q^{2}\right) & =\frac{i}{4 x \pi P^{+}} \int d \xi^{-} e^{-i x \xi^{-} P^{+}}\left\langle P, \hat{e}_{3}\left|\operatorname{Tr}\left\{F^{+\alpha}\left(\xi^{-}, \overrightarrow{0}\right) \mathcal{I}\left(\xi^{-}, 0\right) \tilde{F}_{\alpha}^{+}(0)\right\}\right|_{Q^{2}} \mid P, \hat{e}_{3}\right\rangle \\
& +(x \rightarrow-x)
\end{aligned}
$$

where $F$ (and $A$ ) are matrices $\left(F \equiv \sum_{a=1}^{8} F^{a} \lambda^{a}\right.$, etc. $) .\left\{F^{a}\right\}$ are in the adjoint, and $\left\{\lambda^{a}\right\}$ are in the triplet representation (with $\operatorname{Tr}\left\{\lambda^{a} \lambda^{b}\right\}=\frac{1}{2} \delta^{a b}$ ). $\xi^{ \pm}, \vec{\xi}^{\perp}$ are light-cone coordinates and $\left(\xi^{-}, \overrightarrow{0}\right)$ denotes the point $\xi^{-}, \xi^{+}=\vec{\xi}^{\perp}=0$. The label $Q^{2}$ is a reminder that the tower of local matrix elements in the Taylor expansion of $F \tilde{F}$ are understood to be renormalized at a factorization scale, $Q^{2}$, and finally $\mathcal{I}$ is the Wilson-line integral,

$$
\mathcal{I}\left(\xi^{-}, 0\right)=\mathcal{P} \exp \left(i g \int_{0}^{\xi^{-}} d y^{-} A^{+}\left(y^{-}, \overrightarrow{0}\right)\right)
$$

The standard parton interpretation follows from eq. (5) if we choose $A^{+}=0$ gauge and introduce the momentum decomposition of the fields $F$ and $\tilde{F}$ quantized at $\xi^{+}=0$.

In order to integrate eq. (5) over $x$ we must study the apparent singularity at $x=0$. Physically, $\Delta g\left(x, Q^{2}\right)$ is not expected to diverge as fast as $1 / x$, so the $\xi^{-}$-integral must vanish as $x \rightarrow 0$. This means that the singularity at $x=0$ is integrable. If $\Delta g$ were found to diverge like $1 / x$ or faster, our analysis would have to be reconsidered. With this in mind, we can interchange the $x$ and $\xi^{-}$integrations and, because $\Delta g$ is symmetric in $x$, we can use the principal value prescription at $x=0, f \frac{d x}{x} e^{-i \alpha x}=-i \pi \varepsilon(\alpha)$. We obtain

$$
\Gamma\left(Q^{2}\right)=\frac{1}{2 P^{+}} \int d \xi^{-} \varepsilon\left(\xi^{-}\right)\left\langle P, \hat{e}_{3}\left|\operatorname{Tr}\left\{F^{+\alpha}\left(\xi^{-}\right) \mathcal{I}\left(\xi^{-}, 0\right) \tilde{F}_{\alpha}^{+}(0)\right\}\right|_{Q^{2}} \mid P, \hat{e}_{3}\right\rangle .
$$

This expression cannot be simplified further unless we choose $A^{+}=0$ gauge.

In $A^{+}=0$ gauge $\mathcal{I}=1$ and $F^{+\alpha}=\frac{\partial}{\partial \xi^{-}} A^{\alpha}$, so we may perform the $\xi^{-}$integration. The terms at $\xi^{-}= \pm \infty$ vanish because the integral in eq. (5) converges when $x=0$. Only the local $\left(\xi^{-}=0\right)$ contribution survives. We choose the rest frame for $P$, and after some algebra we are left with the expression we seek,

$$
\begin{aligned}
\Gamma\left(Q^{2}\right) & =\frac{1}{\sqrt{2} M}\left\langle P, \hat{e}_{3}\left|2 \operatorname{Tr}\left\{A^{1} F^{+2}-A^{2} F^{+1}\right\}\right|_{Q^{2}} \mid P, \hat{e}_{3}\right\rangle \\
& =\frac{1}{2 M}\left\langle P, \hat{e}_{3}\left|2 \operatorname{Tr}\left\{(\vec{E} \times \vec{A})^{3}+\vec{A}_{\perp} \cdot \vec{B}_{\perp}\right\}\right|_{Q^{2}} \mid P, \hat{e}_{3}\right\rangle,
\end{aligned}
$$

where $E^{i}=F^{i 0}$, and $B^{i}=-\frac{1}{2} \varepsilon^{i j k} F^{j k}$. The choice of $A^{+}=0$ gauge was essential to this derivation. Without it, $\Gamma$ does not appear to be associated with a local operator.

$A^{+}=0$ does not completely fix the gauge in QCD. Residual non-abelian gauge transformations, $\delta A^{\mu}=\partial^{\mu} \delta \alpha+\left[\delta \alpha, A^{\mu}\right]$, are allowed provided they obey $\partial^{+} \delta \alpha=0$ which preserves $A^{+}=0 . \Gamma$ must be invariant under this residual gauge symmetry. Using the Bianchi identity - $\left[D^{\mu}, F^{\nu \lambda}\right]+\left[D^{\nu}, F^{\lambda \mu}\right]+\left[D^{\lambda}, F^{\mu \nu}\right]=0$ - it is straightforward to show that 


$$
\delta \Gamma \propto\left\langle P, \hat{e}_{3}\left|\operatorname{Tr}\left\{\alpha \partial^{+} F^{12}\right\}\right| P, \hat{e}_{3}\right\rangle
$$

which vanishes because $\alpha$ is independent of $\xi^{-}$and the + derivative sits inside a forward matrix element. Thus $\Gamma$ is invariant under the residual gauge symmetries of $A^{+}=0$ gauge.

Eq. (8) is not a familiar representation for the spin angular momentum stored in a gauge vector field. Happily it can be related directly to the gluon spin term in the angular momentum tensor density in QCD. Angular momentum in QCD, as in any field theory, is described by a rank-3 Lorentz tensor, $M^{\mu \nu \lambda}$. In a physical gauge, where there are no ghosts,

$$
\begin{aligned}
M_{Q C D}^{\mu \nu \lambda} & =\frac{i}{2} \bar{\psi} \gamma^{\mu}\left(x^{\lambda} \partial^{\nu}-x^{\nu} \partial^{\lambda}\right) \psi+\frac{1}{2} \epsilon^{\mu \nu \lambda \sigma} \bar{\psi} \gamma_{\sigma} \gamma_{5} \psi \\
& -2 \operatorname{Tr}\left\{F^{\mu \alpha}\left(x^{\nu} \partial^{\lambda}-x^{\lambda} \partial^{\nu}\right) A_{\alpha}\right\}+2 \operatorname{Tr}\left\{F^{\mu \lambda} A^{\nu}+F^{\nu \mu} A^{\lambda}\right\} \\
& -\frac{1}{2} \operatorname{Tr} F^{2}\left(x^{\nu} g^{\mu \lambda}-x^{\lambda} g^{\mu \nu}\right),
\end{aligned}
$$

The second term in eq. (10) measures the quark spin - at least up to subtleties arising from the triangle anomaly. 5 \& The first and third terms look like the quark and gluon orbital angular momentum respectively, because they have the standard "convective" form of orbital angular momentum in a field theory, $\Pi^{\dagger}(\vec{x} \times \vec{\nabla}) \Phi$, where $\Pi$ and $\Phi$ are canonical coordinate and momentum respectively. The last term contributes only to boosts. The fourth term is a candidate for the gluon spin. 2 Let us define,

$$
M_{\Gamma}^{\mu \nu \lambda} \equiv 2 \operatorname{Tr}\left\{F^{\mu \nu} A^{\lambda}+F^{\lambda \mu} A^{\nu}\right\} .
$$

Then comparison with eq. (8) shows that

$$
\Gamma\left(Q^{2}\right)=\frac{1}{2 S^{+}}\left\langle P, \hat{e}_{3}\left|M_{\Gamma}^{+12}\right|_{Q^{2}} \mid P, \hat{e}_{3}\right\rangle
$$

in $A^{+}=0$ gauge. This identification makes physical sense since the parton model distribution should measure helicity along the $\hat{e}_{3}$-axis (hence $\nu=1, \lambda=2$ ) in an infinite momentum frame, which corresponds to $\mu=+$ in the laboratory. The restriction to $A^{+}=0$ gauge is natural in the parton model. Needless to say, this restriction does not render $\Gamma$ gauge dependent: There is a corresponding operator definition of $\Gamma$ in any gauge. However it is not simple or even local. Note that this definition of gluon spin does not correspond to the gluon piece of the generator of rotations in the laboratory, which would be $M_{\Gamma}^{012}$ and would not naturally appear in light-cone gauge.

This discussion suggests that a natural definition of the quark and gluon orbital angular momentum might select the +12 component of the appropriate piece of $M^{\mu \nu \lambda}$ in eq. (10). (13) While this is attractive, a physically interesting $L_{Q}$ or $L_{G}$ must be determined by what can be measured experimentally. As long as no measure of orbital angular momentum is experimentally accessible, the definition will remain open to question.

\footnotetext{
${ }^{2}$ Eq. (10) was derived in Ref. [7], where the generator of gluon spin rotations, $M_{\Gamma}^{0 i j}$ was incorrectly identified as $\vec{A} \times \vec{E}$. Since this sign is crucial to our results we have checked a) that canonical tranformations give $\vec{E} \times \vec{A}$ and b) that the two gluon contributions combine to give a total angular momentum of $\vec{J}=\vec{x} \times \vec{E} \times \vec{B}$ as expected.
} 


\section{THE GLUON SPIN IN QUARK MODELS}

Quark models of the light hadrons fall into two general classes: non-relativistic quark models, where quarks are described by the Schroedinger equation (perhaps including relativistic corrections) and confined by some two body color dependent forces; and bag models, where relativistic quarks, governed by the Dirac equation, move in some confining background field imagined to be self-consistently generated by their deformation of the nonperturbative QCD vacuum. Both extremes give good explanations of the mass spectrum of the lightest hadrons (pseudoscalar and vector mesons, and octet and decuplet baryons). A major role is played by color mediated, spin dependent forces. 14.15 In this Section we shall see that the gluons responsible for these spin splittings are anti-aligned with the nucleon spin $(\Gamma<0)$; that this is a particular consequence of the non-abelian nature of QCD interactions, and that the effect has roughly the same magnitude in both types of model.

The quantity we want to evaluate in models is

$$
\Gamma\left(\mu_{0}^{2}\right)=\left\langle T, \hat{e}_{3}\left|\int d^{3} x 2 \operatorname{Tr}\left\{(\vec{E}(\vec{x}) \times \vec{A}(\vec{x}))^{3}+\vec{A}_{\perp}(\vec{x}) \cdot \vec{B}_{\perp}(\vec{x})\right\}\right| T, \hat{e}_{3}\right\rangle
$$

One way to evaluate this expression would be to compute the relevant Feynman diagram - obtained by inserting this gluonic operator into the lowest order Born diagram for gluon exchange between bound quarks (in $A^{+}=0$ gauge). The graphical method would require us to construct and use confined gluon Green's functions in $A^{+}=0$ gauge, which is unnecessarily complicated. It is easier to compute the ambient color fields by directly integrating the QCD equations of motion (which reduce to eight copies of Maxwell's equations in the Abelian approximation). In this way we obtain expressions for $\vec{A}, \vec{B}$, and $\vec{E}$ which depend on the quark color, spin and spatial coordinates and can be evaluated with the help of model wavefunctions.

In a generic quark model the color-electric fields will be given by the gradient of a timeindependent function of the quark degrees of freedom, $\vec{E}^{a}(\vec{x})=-\vec{\nabla} \Phi^{a}(\vec{x})$, with

$$
\Phi^{a}(\vec{x})=\frac{g}{4 \pi} \sum_{i} \lambda_{i}^{a} \mathcal{G}\left(\vec{x}, \vec{x}_{i}\right)
$$

$\Phi$ is an operator in the space of the quark color $\left(\lambda_{i}^{a}\right)$ and position $\left(\vec{x}_{i}\right)$ states. It depends on some model Green's function $\mathcal{G}$. For example, in an unconfined, non-relativistic model $\mathcal{G}=1 /\left|\vec{x}-\vec{x}_{i}\right|$.

The magnetic field, $\vec{B}^{a}(\vec{x})$ can likewise be written as the curl of a time independent function of the quark variables, $\vec{B}^{a}(\vec{x})=\vec{\nabla} \times \vec{U}^{a}(\vec{x})$, with

$$
\vec{U}^{a}(\vec{x})=\frac{g}{4 \pi} \sum_{i} \lambda_{i}^{a} \vec{\sigma}_{i} \times \overrightarrow{\mathcal{G}}\left(\vec{x}, \vec{x}_{i}\right)
$$

\footnotetext{
${ }^{3}$ Quark model states are more conveniently normalized to unity than covariantly, so we change normalization accordingly, $\langle P|\mathcal{O}(0)| P\rangle=2 M \int d^{3} x\langle T|\mathcal{O}(\vec{x})| T\rangle$, where $|T\rangle$ is a quark model state normalized to unity.
} 
For example, $\overrightarrow{\mathcal{G}}=\left(\vec{x}-\vec{x}_{i}\right) / 2 m_{q}\left|\vec{x}-\vec{x}_{i}\right|^{3}$ in a non-relativistic model (where $\vec{m}_{i}^{a} \equiv g \vec{\sigma}_{i} \lambda_{i}^{a} / 2 m_{q}$ is the quark's color magnetic moment operator). The nucleon polarization (and the gauge choice, $\left.A^{+}=0\right)$ selects the $\hat{e}_{3}$-axis. Matrix elements of the operator $\vec{\sigma}_{i}$ will therefore vanish except in the $\hat{e}_{3}$ direction. So we conclude that $\left\langle\vec{U}_{i}^{a} \cdot \hat{e}_{3}\right\rangle=\left\langle U_{i}^{a 3}\right\rangle=0$.

So far this result is quite general. It holds in any quark model where the gluons are treated to lowest order (abelian approximation) and $\vec{U}$ has no component along the nucleon spin. In "symmetric" quark models the quark distributions in the nucleon ground state are not correlated with the overall spin. When integrated over the wavefunction of the $j^{\text {th }}$ quark, then, the resulting color electric field is radial and independent of $j$,

$$
-\int d^{3} x_{j} \vec{\nabla} \mathcal{G}\left(\vec{x}, \vec{x}_{j}\right)\left|\psi\left(\vec{x}_{j}\right)\right|^{2}=\frac{\vec{x}}{r^{3}} Q(r),
$$

where $r=|\vec{x}|$ and $Q(r)=4 \pi \int_{0}^{r} d r^{\prime} r^{\prime 2}\left|\psi\left(r^{\prime}\right)\right|^{2}$ is the color charge inside the sphere with radius $r$. Likewise integration over $\vec{x}_{i}$ simplifies $\overrightarrow{\mathcal{G}}$,

$$
\int d^{3} x_{i} \overrightarrow{\mathcal{G}}\left(\vec{x}, \vec{x}_{i}\right)\left|\psi\left(\vec{x}_{i}\right)\right|^{2}=\vec{x} h(r)
$$

where $\mathrm{h}(\mathrm{r})$ describes the vector potential generated by the model dependent magnetization density.

The operators $\Phi$ and $\vec{U}$ are not yet the appropriate scalar and vector potentials for the gluon field because they do not satisfy the $A^{+}=0$ gauge condition. However suitable potentials are easily constructed. Define

$$
\begin{aligned}
A^{0 a}(\vec{x}) & =\Phi^{a}(\vec{x}) \\
\vec{A}^{a}(\vec{x}) & =\vec{U}^{a}(\vec{x})-\vec{\nabla} \int_{0}^{z} d \zeta \Phi^{a}(x, y, \zeta)
\end{aligned}
$$

These potentials generate $\vec{E}^{a}$ and $\vec{B}^{a}$ in the usual way and satisfy the gauge constraint, $A^{0 a}+A^{3 a}=0$ (remembering $U^{3 a}=0$ ). The choice of time independent potentials as well as the lower limit on the $\zeta$ integration correspond to residual gauge freedom available in $A^{+}=0$ gauge.

We now substitute the operator definitions of $A^{0 a}$ and $\overrightarrow{A^{a}}$ into eq. (13) and obtain

$$
\begin{aligned}
\Gamma\left(\mu_{0}^{2}\right) & =\sum_{i \neq j} \sum_{a=1}^{8} \int d^{3} x\left\langle T, \hat{e}_{3}\right|\left\{\left[\vec{E}_{i}^{a}(\vec{x}) \times \vec{U}_{j}^{a}(\vec{x})\right]^{3}+\left[\vec{E}_{i}^{a}(\vec{x}) \times \vec{\nabla} f_{j}^{a}(\vec{x})\right]^{3}\right. \\
& \left.+\vec{U}_{\perp i}^{a}(\vec{x}) \cdot \vec{B}_{\perp j}^{a}(\vec{x})+\vec{\nabla}_{\perp} f_{i}^{a}(\vec{x}) \cdot \vec{B}_{\perp j}^{a}(\vec{x})\right\}\left|T, \hat{e}_{3}\right\rangle
\end{aligned}
$$

where we have separated out the contributions from individual quarks $i$ and $j$ to each of the field operators. Note we have dropped the $i=j$ terms. These correspond to "selfangular momentum" effects and are associated with questions of renormalization, which are discussed further in Section 4.

The second and third terms in eq. (19) vanish. The second vanishes after integration by parts because $\vec{\nabla} \times \vec{E}_{j}^{a}=0$. There is no associated surface term. The third term vanishes for spatially symmetric quark wavefunctions.

Consider now the fourth term and write out the space components explicitly (suppressing the color $(a)$ and quark $(i, j)$ labels, and bras and kets, 


$$
\begin{aligned}
\Gamma_{4} & =\int d^{3} x\left(\frac{\partial f}{\partial y} \frac{\partial U_{1}}{\partial z}-\frac{\partial f}{\partial x} \frac{\partial U_{2}}{\partial z}\right) \\
& =\int d^{3} x\left(E_{1} U_{2}-E_{2} U_{1}\right) \\
& +\oint_{S_{R}} d^{2} s \hat{e}_{3} \cdot \hat{r}\left(U_{1}(\vec{x}) \int_{0}^{z} d \zeta E_{2}(x, y, \zeta)-U_{2}(\vec{x}) \int_{0}^{z} d \zeta E_{1}(x, y, \zeta)\right)
\end{aligned}
$$

where the surface integral is over a sphere at large distance (for unconfined gluons) or the bag surface (for bag-like models). The first term in eq. (20) is identical to the first term in eq. (19).

We combine these results and substitute the parameterizations of $\vec{E}$ and $\vec{U}$ from eq's. (16) and (17) to obtain,

$$
\Gamma=\frac{8}{9} \alpha \int_{0}^{R} d r r Q(r)(h(R)-2 h(r)),
$$

where the $r$-integration goes to infinity in generic non-relativistic quark models, but ends at $r=R$, the bag surface in the bag model. The term proportional to $-2 h(r)$ is the volume integral of $\vec{E} \times \vec{U}$, and the $h(R)$-term is the surface contribution left over from integration by parts. In reaching eq. (21) we have used $\sum_{a=1}^{8} \lambda_{i}^{a} \lambda_{j}^{a}=-2 / 3$ for $i \neq j$. [Because the nucleon is a color singlet, $\left[\sum_{a=1}^{8} \sum_{j=1}^{3} \lambda_{j}^{a}\right]^{2}=0$. The result follows since $\sum_{a=1}^{8}\left(\lambda_{j}^{a}\right)^{2}=4 / 3$.] Also, $\alpha=g^{2} / 4 \pi$ and $\sum_{i \neq j}\left\langle\sigma_{i}^{3}\right\rangle=2$ in a state polarized along the $\hat{e}_{3}$-direction.

Now let us specialize to quark potential models where quarks are confined but color is not. Gluon field strengths fall off at large distances like abelian multipoles. The nonrelativistic vector potential, $\vec{U}=\vec{m} \times \vec{r} / r^{3}$, corresponds to $h(r)_{N Q M} \propto 1 / 2 m_{q} r^{3}$. At large $R, Q(R) \rightarrow 1$, so the surface term in eq. (21) vanishes. Substituting for $Q(r)$ in terms of the quark wavefunction, $\psi(r)$ and interchanging integrations, eq. (21) reduces to

$$
\begin{aligned}
\Gamma_{N Q M}\left(\mu_{0}^{2}\right) & =-\frac{8}{9 m_{q}} \alpha_{N Q M}\left(\mu_{0}^{2}\right) \int_{0}^{\infty} d r r|\psi(r)|^{2} \\
& =-\frac{8}{9 m_{q}} \alpha_{N Q M}\left(\mu_{0}^{2}\right)\left\langle\frac{1}{r}\right\rangle,
\end{aligned}
$$

where we have restored the quark model renormalization scale, $\mu_{0}^{2}$, to remind us that this value pertains to some low scale at which the model is formulated.

The parameters $m_{q},\left\langle\frac{1}{r}\right\rangle$ and $\alpha_{N Q M}\left(\mu_{0}^{2}\right)$ are all model dependent, but not unconstrained. $m_{q} \approx 0.3 \mathrm{GeV}$ reproduces nucleon magnetic moments. Another constraint comes from the $\Delta-N$ mass difference which is given by

$$
\Delta M=\frac{8 \pi}{3} \frac{\alpha_{N Q M}\left(\mu_{0}^{2}\right)}{m_{q}^{2}}\left\langle\delta^{3}(\vec{r})\right\rangle
$$

in the non-relativistic quark model. 14] To obtain a numerical estimate, we assume a gaussian wavefunction adjusted to reproduce the root-mean-square charge radius of the proton. Then we find $\alpha_{N Q M}\left(\mu_{0}^{2}\right) \approx 0.9$, and $\Gamma_{N Q M}\left(\mu_{0}^{2}\right) \approx-0.8$.

Static bag model calculations of hadron spin splittings were carried out by explicit construction of color electric and magnetic fields. 15 We may borrow results from that work 
to evaluate eq. (21). The color magnetic field is calculated from the QCD generalization of Maxwell's equation, $\vec{\nabla} \times \vec{B}^{a}=g \psi^{\dagger} \vec{\alpha} \lambda^{a} \psi$ augmented by the boundary condition $\hat{r} \times \vec{B}^{a}=0$ at $r=R$. A short calculation yields,

$$
\begin{aligned}
Q(r) & =\int_{0}^{r} d r^{\prime} r^{\prime 2}\left(f^{2}\left(r^{\prime}\right)+g^{2}\left(r^{\prime}\right)\right) \\
h(r) & =\left\{\frac{1}{2} \frac{\mu(R)}{R^{3}}+\frac{\mu(r)}{r^{3}}+\int_{r}^{R} d r^{\prime} \frac{\mu^{\prime}\left(r^{\prime}\right)}{r^{\prime 3}}\right\}, \quad \text { where } \\
\mu(r) & =\int_{0}^{r} d r^{\prime} \frac{8 \pi}{3} r^{3} f(r) g(r)
\end{aligned}
$$

where $f(r) \propto j_{0}\left(x_{0} r / R\right)$ and $g(r) \propto j_{1}\left(x_{0} r / R\right) .\left[x_{0}\right.$ is the lowest solution to the eigenvalue condition $\tan x=x / 1-x \quad\left(x_{0}=2.0428\right)$.] Substituting explicit wavefunctions we find

$$
\Gamma_{b a g}\left(\mu_{0}^{2}\right)=-0.1 \alpha_{b a g}\left(\mu_{0}^{2}\right)
$$

Standard bag model calculations of baryon spin splittings require $\alpha_{Q C D} \approx 2$, so we find $\Gamma_{b a g} \sim-0.2$ at the renormalization scale of the model.

\section{DISCUSSION AND CONCLUSIONS}

Our calculations force us to conclude that the gluons responsible for the spin splittings among light baryons are anti-aligned with the spin of their parent nucleon. This exacerbates rather than helps resolve the problem with the nucleon spin, suggesting we look elsewhere for a large positive contribution to the spin. Our calculation required two assumptions beyond the traditional formulation of quark models: first, that "self-interaction" contributions to $\Gamma$ could be ignored, and second, that the estimate of $\Gamma$ at the quark model scale, $\mu_{0}^{2}$ has something to do with its value at experimentally accessible scales. Here we will first look at the reliability of our estimate in the context of the models. Then we will comment further on the two assumptions and finally mention some possible extensions of the work.

The troubling sign of $\Gamma$ does not appear to depend on the details of the models. The directions of the color electric and magnetic fields are fixed by the charges and currents which give successful descriptions of a variety of hadronic phenomena including magnetic moments as well as colormagnetic spin splittings. The minus sign arises because the gluons are non-abelian - the two spectators of any given quark in the nucleon generate color fields appropriate to an antiquark. The same effect flips the sign of baryon spin splittings. If quark spin forces were abelian, $\Gamma$ would be positive, but the $\Delta$ would be lighter than the nucleon.

On the other hand, the magnitude is of $\Gamma$ quite uncertain. The value of $\Gamma\left(Q^{2}\right)$ at scales relevant to experiment varies quadratically with the assumed value of $\alpha\left(\mu_{0}^{2}\right)$ taken from fits to baryon spin splittings. One factor of $\alpha$ comes from the operator itself; the other factor arises in scaling from $\mu_{0}^{2}$ to $Q^{2}$. There is reason to believe that symmmetric quark models overestimate the value of $\alpha$ necessary to account for spin splittings because they ignore the correlations color magnetic interactions would introduce into wavefunctions. We expect that the color magnetism would correlate quark pairs into color $\overline{3}$, spin 0 diquarks (the most attractive channel) with the effect of increasing the matrix element and decreasing 
the value of $\alpha$ necessary to reproduce spin splittings. An estimate of the size of this effect goes beyond the scope of this Letter. However we caution the reader against taking the calculated magnitude of $\Gamma$ very seriously. The most important conclusion of our work is the prediction that the sign of $\Gamma$ should be negative. If experiment finds $\Gamma$ to be positive, and if neither self-interactions nor evolution change the model predictions, then the quark model calculations of baryon spin splittings will have to be re-evaluated.

Regarding the self interaction terms, the proper prescription in principle is "calculate all graphs and renormalize". Consider the case of an isolated electron in QED: the $\vec{E}$ and $\vec{B}$ fields which surround it contribute a log-divergent term to the expectation of $M_{\Gamma}^{+12}$. The same occurs for the stress tensor (one piece of which is the self-energy). Renormalization removes these infinities. Since $M_{\mu \nu \lambda}=x_{\mu} T_{\nu \lambda}-x_{\nu} T_{\mu \lambda}$ (up to a total derivative) renormalizing $T$ also renormalizes $M$. If we renormalize on-shell, the spin is found on the renormalized electron line and the self-fields are to be ignored. The same argument would seem to carry over to the quark model and dictate that we ignore all terms with $i=j$ in eq. (13). However we cannot renormalize a confined quark on mass-shell, so we cannot assume that all selffield effects can be renormalized away. The problem also arises in the bag model where the calculation and renormalization of the self-field contribution to $M_{\Gamma}^{+12}$ would yield a finite result analogous to the Lamb shift correction to the self-energy of a bound electron. This program has actually been carried out for the self-energy of a quark in a bag. 16 . Only a complete treatment of the renormalization of a confined quark would enable one to decide how much of the self-field should be included in the normalization of a quark at the scale $\mu_{0}^{2}$ and how much should be attributed to its binding inside the nucleon. We have, in effect, assumed that all self-field effects are to be included in the definition of the renormalized quark operators and omitted from our calculation of $\Gamma$. This assumption should be examined more closely; it is possible that finite parts of the $i=j$ terms make a significant contribution to $\Gamma$ at the scale $\mu_{0}^{2}$.

The renormalization point and scheme dependence of $\Gamma$ has been discussed extensively. In $A^{+}=0$ gauge it is easy to see that the forward matrix element of the Kogut-Susskind current, $17\left\langle K^{+}\right\rangle$, coincides with our definition of $\Gamma$. So the studies of the QCD evolution of the forward matrix elements of $\left\langle K^{+}\right\rangle\left[\begin{array}{l}{[18]} \\ {[1}\end{array}\right.$ apply without alteration to the evolution of $\Gamma$. To leading order $\alpha_{Q C D}\left(Q^{2}\right) \Gamma\left(Q^{2}\right)$ is independent of $Q^{2}$. Beyond leading order the $Q^{2}$ dependence of $\Gamma$ is scheme dependent, and not of much interest until one understands the renormalization scale and scheme dependence of quark model calculations. These problems notwithstanding, it appears that the quark model result $\Gamma<0$ is preserved by leading order evolution.

The analysis presented here raises several questions and suggests some avenues for future study. First, no doubt, is to understand better the origin of the result $\Gamma_{N Q M}<0$ and its generality. The light-cone perturbation theory formalism pioneered by Brodsky and Lepage, 19$]$ and applied to the parton structure of positronium by Burkardt, [2] seems like the natural framework in which to explore the result further. Another direction would be to use these models to explore the $x$-dependence of the nucleon's polarized gluon distribution (at the scale $\mu_{0}^{2}$ ), since the shape of $\Delta g$ is of interest. 21] These calculations might serve to guide the application of sophisticated (next-to-leading order) perturbative QCD fits to spin dependent gluon distributions. ${ }^{22}$ Finally we note that there is much less reason to compute the spin-independent gluon distribution using these models. The lowest moment of $g\left(x, Q^{2}\right)$ 
does not converge and is not associated with a local operator. Furthermore models that take seriously the gluon role in confinement, leave us puzzled whether the long-range confining

field (e.g. the bag itself in the bag model) should be associated with a (spin-independent) gluon distribution or not.

\section{ACKNOWLEDGEMENTS}

I would like to thank the organizers and participants in the 1995 Erice School on Nucleon Spin Structure where this work was conceived, for discussions, and the Physics Department at Harvard University, where it was performed, for hospitality. 


\section{REFERENCES}

[1] J. Ashman et al. Phys. Lett. B206, 364 (1988); Nucl. Phys. B328, 1 (1989).

[2] For a review and summary of experimental data and further references, see C. Cavata and V. Hughes, eds. Proceedings of the Yale Workshop (Plenum Press, New York, 1995).

[3] L. M. Sehgal, Phys. Rev. D10, 1663 (1974).

[4] J. Ellis and R. L. Jaffe, Phys. Rev. D9, 1444 (1974), D10, 1669 (1974).

[5] A. V. Efremov and O. V. Teryaev, Czech. Hadron Symposium (1988), 302; G. Alterelli and G. G. Ross, Phys. Lett. B212 (1988) 391.

[6] R. D. Carlitz, J. C. Collins and A. H. Mueller, Phys. Lett. B214 (1988) 229.

[7] R. L. Jaffe and A. Manohar, Nucl. Phys. B337 (1990) 509;

[8] G. Bodwin and J. Qiu, Phys. Rev. D41 (1990) 2755.

[9] J. C. Collins and D. E. Soper, Nucl. Phys. B194 445 (1982); A. Manohar, Phys. Rev. Lett. 65 (1990) 2511, ibid. 66 (1991) 289.

[10] Dynamics of the Standard Model By J. F. Donoghue, E. Golowich, and B. R. Holstein, (Cambridge University Press, Cambridge, 1992).

[11] Models of the Nucleon By R. K. Bhaduri, (Addison Wesley, Reading, 1988).

[12] G. Altarelli, Phys. Rep. 81 (1982) 1.

[13] P. Hoodbhoy, X. Ji, and J. Tang, MIT-CTP-2476, hep-ph/9510304, 1995.

[14] A. de Rujula, H. Georgi and S. L. Glashow, Phys. Rev. D12 147 (1975).

[15] T. A. DeGrand, R. L. Jaffe, K. Johnson, and J. Kiskis, Phys. Rev. D12 2060 (1975).

[16] S. N. Goldhaber, T. H. Hansson, and R. L. Jaffe, Phys. Lett. 131B (1983) 445, Nucl. Phys. B277 (1986) 674

[17] J. Kogut and L. Susskind, Phys. Rev. D11, 3594 (1974).

[18] J. Kodaira, Hiroshima University preprint, HUPD-9405 (January 1995), hep-ph 9501381, and references therein.

[19] S. J. Brodsky and G. P. Lapage, Phys. Rev. D22, 2157 (1980).

[20] M. Burkardt, Nucl. Phys. B373 (1992) 371.

[21] R. Jaffe, Phys. Rev. D11 (1975) 1953; R. Jaffe and X. Ji, Phys. Rev. D43 (1991) 724;

M. Stratmann, Z. Phys. C60 (1993), 763.

[22] M. Glück, E. Reya, and A. Vogt, Z. Phys. C67 (1995) 433, and references therein. 